HIFAN 1820

\title{
Time Delays, Bends, Acceleration and Array Reconfigurations
}

\author{
by \\ Andris Faltens \\ from \\ Accelerator and Fusion Research Division \\ Ernest Orlando Lawrence Berkeley National Laboratory \\ Berkeley, California 94720
}

November 2010

This work was supported by the Director, Office of Science, Office of Fusion Energy Sciences, of the U.S. Department of Energy under Contract No. DE-AC02-05CH11231. 
This document was prepared as an account of work sponsored by the United States Government. While this document is believed to contain correct information, neither the United States Government nor any agency thereof, nor The Regents of the University of California, nor any of their employees, makes any warranty, express or implied, or assumes any legal responsibility for the accuracy, completeness, or usefulness of any information, apparatus, product, or process disclosed, or represents that its use would not infringe privately owned rights. Reference herein to any specific commercial product, process, or service by its trade name, trademark, manufacturer, or otherwise, does not necessarily constitute or imply its endorsement, recommendation, or favoring by the United States Government or any agency thereof, or The Regents of the University of California. The views and opinions of authors expressed herein do not necessarily state or reflect those of the United States Government or any agency thereof or The Regents of the University of California. 


\title{
Time Delays, Bends, Acceleration and Array Reconfigurations
}

by

\author{
Andris Faltens
}

This note was originally one of the parts of the work on a $50 \mathrm{MeV}$ and $500 \mathrm{MeV} \mathrm{Rb^{+ }}$

driver (1) and part of work on delay lines for a $60 \mathrm{GeV} \mathrm{U}^{+12}$ driver. (2) It is slightly expanded here to make it more generally applicable. The emphasis is on beam manipulations such as joining and separating beams at the two ends of a driver and providing various time delays between beams as required by the target.

\section{Introduction}

In various HIF scenarios, a large number of beams is transported and accelerated in parallel because of much lower cost. At the front of the accelerator it is necessary to bring these beams together into a compact array, and at the end of the accelerator the beams must be separated to allow groups of beams to be individually focused to impose the desired irradiation symmetry onto the target. In addition, shaped pulses usually are required for matching the input power to the capsule implosion, a time of some $30 \mathrm{~ns}$ in standard targets and about $120 \mathrm{~ns}$ in some recent work. The power shaping could be attained by changing the time profile of each individual pulse or by a piecewise linear process of using several groups of pulses which are delayed as desired. The focusing of a shaped pulse, with almost inevitable energy and current variations during the pulse is clearly a more difficult process than focusing groups of substantially constant current and monoenergetic pulses, and that is the emphasis in this note.

The reasons for using compact arrays of beams are easy to understand. A single beam, whether in an electrically or magnetically focused quadrupole channel requires electrodes or coils around it to generate the field. In most cases there is a substantial field outside of the electrodes. As an example, one electrostatic quadrupole of the high-voltage type requires four electrodes, and has external field, which must be included in the design. A large array of these quadrupoles, however, approaches only one electrode per beam, as each electrode can be shared by four beams, and there is no unused field except at the edges of the array. The magnetic case is completely analogous. The rest of the induction accelerator, that is, the insulators and induction core become correspondingly smaller.

The standard driver concept involves bringing beams together at low energy and separating them at high energy. In particular, many driver concepts involve separating groups of beams at various energies to provide one form of pulse shaping at the target, the other being shaping of the energy distribution within each pulse by appropriately designed waveforms. Beam merging has already been done at a small scale in the beam merging experiment, where four beams were made to coalesce into one beam, and the resultant emittance growth was as expected. Changing the distance between beams is a simpler operation and does not result in significant emittance dilution. At low energies, the basic situation is that the transport is the limiting item, and just to transport the beams requires an almost unbroken line of electrostatic quadrupoles, with a longitudinal occupancy $\eta$ of about $80 \%$. While quadrupoles stack nicely into an efficient and compact array, electric dipoles do not seem to. Instead of using dipoles and quadrupoles to bend the low energy beams, the bending may be achieved by slightly displaced quadrupoles. A displaced quadrupole of gradient $\mathrm{E}^{\prime}=\mathrm{dE} / \mathrm{dx}=2 \mathrm{~V} / \mathrm{r}$, where $\mathrm{V}$ is the voltage on an electrode and $\mathrm{r}$ is the radius from the center of the channel to the electrode, will produce a displaced quadrupole of the same gradient and a dipole of strength $\mathrm{Ed}=\mathrm{E}^{\prime} \delta$ for a displacement $\delta$. Because the quadrupoles alternate from $\mathrm{F}$ to $\mathrm{D}$ along the lattice, the displacements must correspondingly 
alternate direction, and this must be done for each axis. There is a previous note on separation of beams with electric quads (1), and joining them is just the same picture with the beam direction reversed. For these reasons, the note focuses on the harder problem of separating substantially identical beams of almost constant energy and current at high energy, rather than the problem of bringing them together at low energy.

At high energies, focusing is usually with magnetic quadrupoles, which have a low occupancy $\eta$ of about $10 \%$ near the output. Previous economics tradeoffs with the program LIACEP showed that the minimum cost driver used moderate fields circa $3 \mathrm{~T}$ instead of the highest fields attainable. To separate closely stacked beams in this situation, one solution is, first, to increase $\eta$ while lowering B, keeping the tune, proportional to $\eta \mathrm{B}$ constant. Then, when the field at the symmetry planes between channels has been reduced to $1.5 \mathrm{~T}$, the safe operating level of iron, to insert sheets or plates of iron at the symmetry planes. The basic cell for electric or magnetic transport is a square in the transverse plane. The coil windings remain the same as before. As the beams are moved apart, the thickness of the iron along the desired separation symmetry line is progressively increased until the point where the iron is sufficiently thick to carry the full external flux. Figure (1) illustrates the basic process. The external flux initially went immediately into the neighboring channel across the dividing line, but ends up being turned towards different neighboring channels as shown. The iron is only needed along the boundaries where beams are separated; the remaining beams maintain their previous dense packing. The required yoke thickness is kept constant after the thickness for full flux is reached as the separation is increased further. In this version of beam separation, weak dipole fields, one up on one side of the separation, one down on the other side, say, are assumed to penetrate the entire array, to obtain the small bends required for the separation. Such fields are obtainable from a picture frame geometry, where there are current sheets on each edge of the array and along the symmetry planes, that is, septum magnets. After separation, these fields can be increased at will to guide the beams until at some point it becomes more economical to alternate quads and dipoles.

A 21 Beam Array illustrating beam orientation and edge windings. Many driver designs require arrays of $\sim 100$ beams or more. The beam separation planes could be chosen along any symmetry lines between the rows or columns of beams.

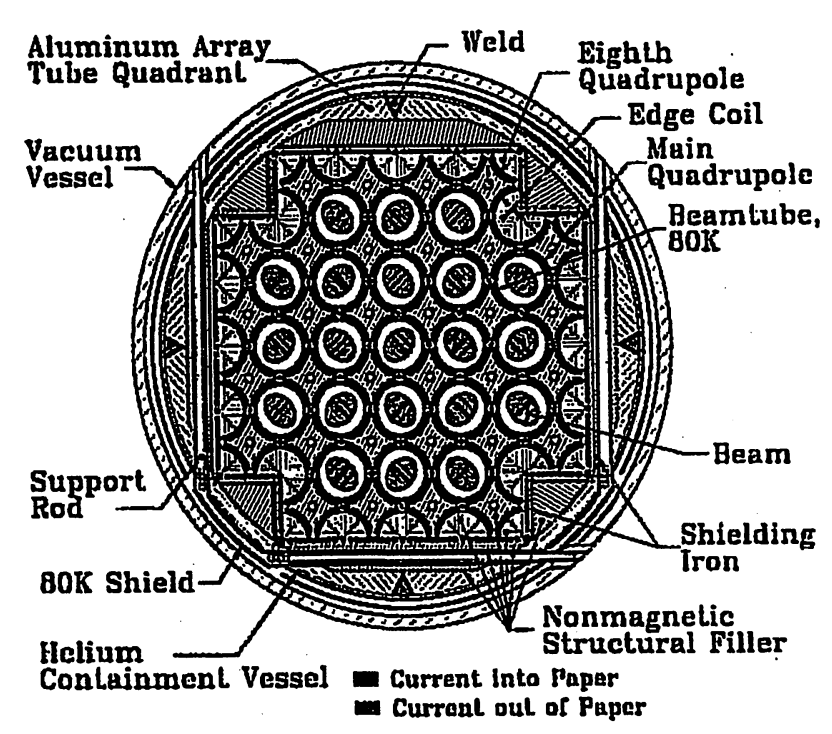



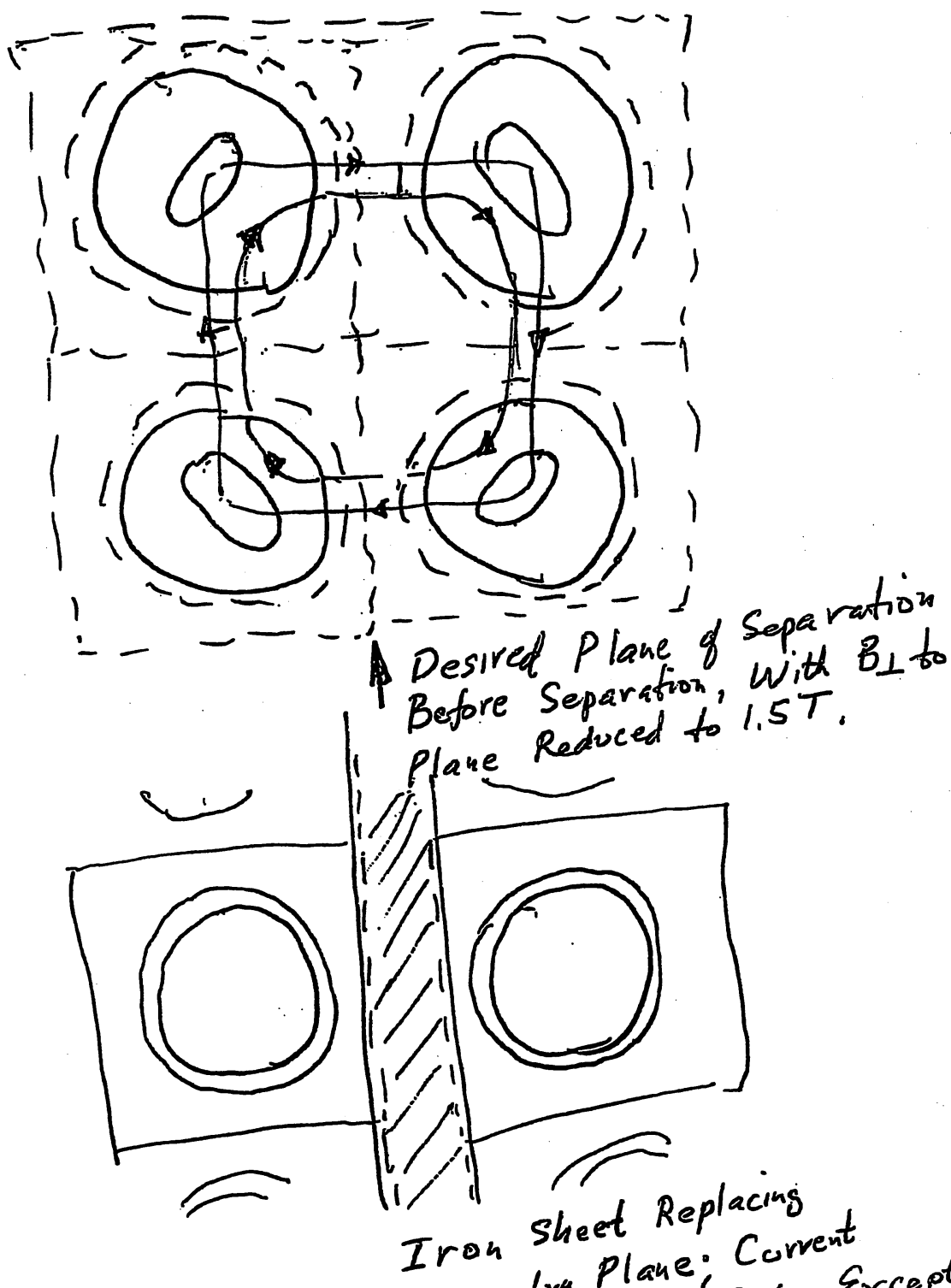

Symmetry Plane: Current

Iron sheet Re pe: Current
Symmetry Plane; Before Except for
Distributions as Before nt Plane
Current Sheets Along Plan
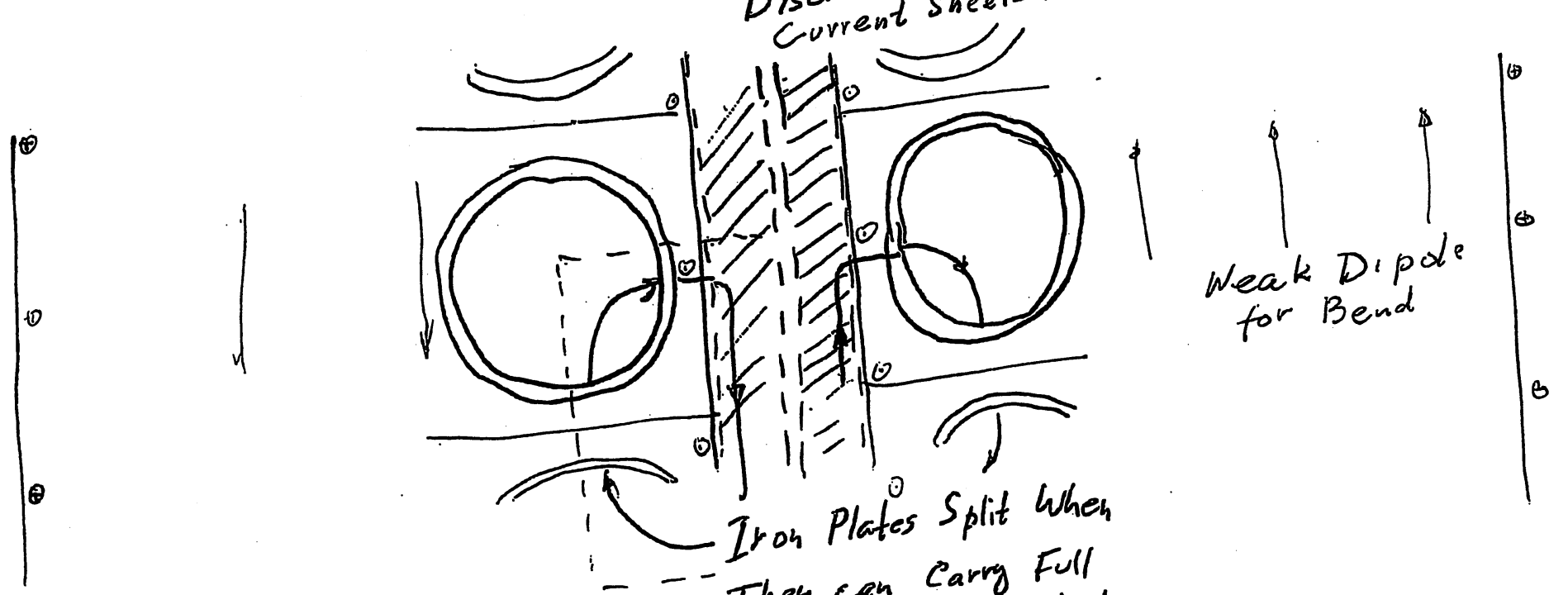

They can Carry Full

Flux of Each set of

Quads 


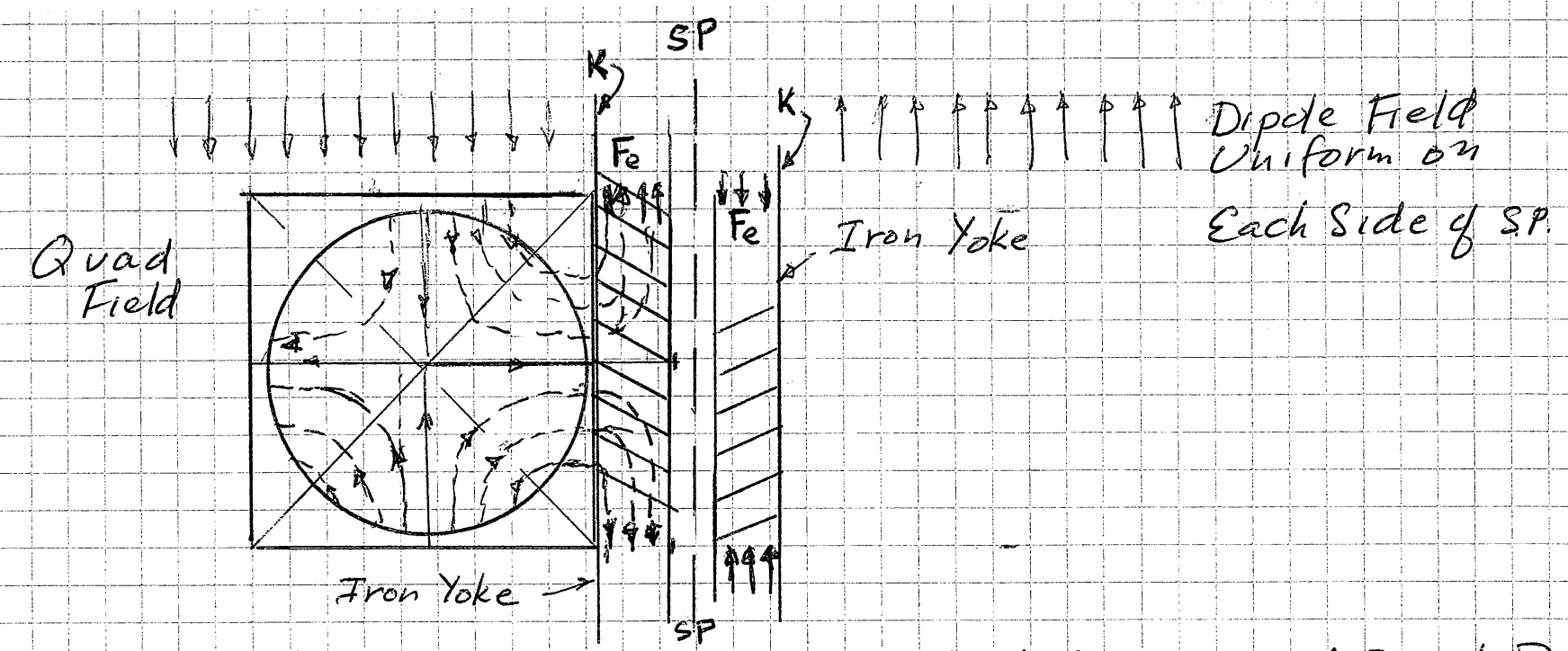

Perfect Magnetic Quad with Superimposed Small Dipole Field Along diagonal, flax $\phi$

is $\frac{(2 w)\left(B_{p}\right)}{2}$. For $B_{p}=B_{F e}=1.5 T$,

all the flux passes through

an iron thickness of $\frac{\sqrt{2} w}{2}$.

$A$ thin current sheet $K$ supplies

the dipole field, B dipole $<<$ B quad

The direction of the field within

the iron reverses sigh across

the symmetry plane SP.

A current sheet of each side

of the array and between each

iron plate and the array 
A recent scenario has four groups of pulses (2), which arrive at the target at different times and an accelerator from which all the pulses leave at the same time with a 30ns duration and a $\pm 3 \%$ velocity tilt. The various required delays may be obtained by speeding up some of the groups, by keeping them all at the same speed but varying their path lengths, or by some combination of these. While having further acceleration might seem inefficient, some means to fine-tune the beams may be necessary to synchronize arrival times to less than the desired pulse duration at the target of 50ps or 100ps.

The chromatic aberration of the semi-final focusing lenses limits the tolerable momentum spread to $\pm 3 \%$ in the present concept and to a few tenths of $1 \%$ in the previous concepts. The tenth percent tolerance range used to translate to $1-2 \%$ waveform tolerances in the accelerator pulsers and $0.1 \%$ in the $\sim 2 \mathrm{MV}$ injector. There used to be efforts to make the old systems more achromatic to ease the pulser requirements.

For clarity and simplicity only the earliest and latest beams are considered for, say, a 120ns delay between groups of $60 \mathrm{GeV} \mathrm{U}^{+12}$ beams.

First, a large tilt is imposed on the beams to aid in producing the slow fast-ignition pulse. At the accelerator exit the $30 \mathrm{~ns}$ bunches are

and the drift compression line is

$$
\ell_{b}=\beta c t=(0.6)\left(3 \times 10^{8}\right)\left(30 \times 10^{-9}\right)=5.4 \text { meters long, }
$$

$$
\ell d=\frac{\ell b}{\frac{\delta \beta}{\beta}}=\frac{5.4}{.06}=90 \text { meters in length. }
$$

The transit time for this line is

$$
\tau=\frac{\ell d}{\beta c}=500 \mathrm{~ns}
$$

Looking at the further-acceleration option first, to have the igniter beams arrive $120 \mathrm{~ns}$ later the first beams are to be accelerated enough to arrive in 380ns. If this acceleration were "instantaneous", the

$$
\begin{aligned}
& \beta \rightarrow \frac{(0.6)(500)}{380}=0.789, \\
& \gamma \rightarrow 1.629 \\
& \Delta \gamma=0.379,
\end{aligned}
$$

or more than then original accelerator provided, so this option in out.

The original accelerator was assumed to provide $2 \mathrm{MV} / \mathrm{m}$. Various combinations of acceleration and pure drift are possible, so the following is an illustration of one such system. Unfortunately, the igniter beam is the one with the stringent quality requirements, and it is that beam which has to take the longest path with bends while at the same time having a large momentum spread. Errors from misalignments are a major concern here, because it is harder to align on an arc than in a straight line, and the anticipated result is wandering of the beam centroids.

Adding an acceleration section of $\sim 200 \mathrm{~m}$ at $2 \mathrm{MV} / \mathrm{m}$ for the fast beam followed by $\sim 350 \mathrm{~m}$ of drift produce about the desired time delay, but such a long drift is expensive and makes the longitudinal emittance requirement harder to achieve than for the path-length-difference system below. 


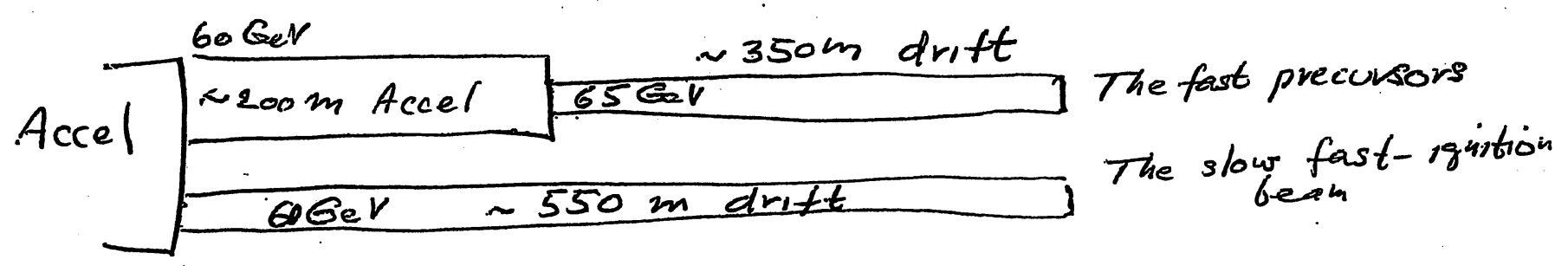

Accelerating some of the beams, followed by drift, to obtain $\sim 120 \mathrm{~ns}$ time delay.

Part of the problem is that $\beta$ changes relatively slowly on a relativistic beam as it is accelerated, and it would make little sense to decelerate any of the beams.

To get the time delays by path-length differences requires bends, which we have tried to avoid.

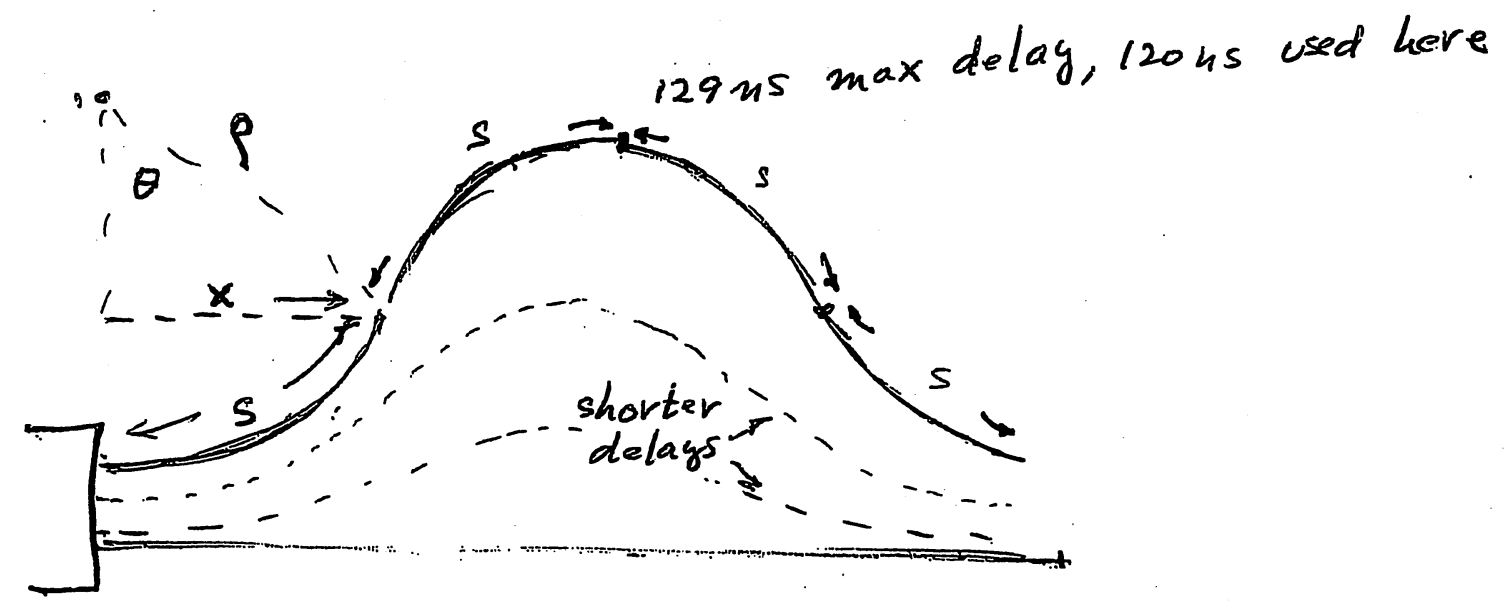

Drift lines with 4 Symmetric Bends to obtain the time delays.

For a radius of the bend $\rho$, and a bend $\theta$ then our length is $s=\rho \theta$ while the distance $\mathrm{x}$ in the original direction of travel is $\rho \sin \theta$. The path length difference for the four necessary arcs is $\Delta=4 \rho(\theta-\sin \theta)$.

To get a delay of $120 \mathrm{~ns}$ at $\beta=0.6$ requires $\Delta=21.6 \mathrm{~m}$.

The bends and focusing in them have not been designed. If the focusing is very frequent and strong, the radial aperture that is required would be similar to that in the straight sections. If the focusing is weak, then the higher energy ions travel at a substantially larger radius than the lower energy ions, and the aperture must be increase. This increase,

$$
\Delta R \approx\left(\frac{\Delta \rho}{\rho}\right) \frac{\rho}{Q^{2}} \text { where } Q \text { is the number of oscillations in a complete circle }
$$

of radius of curvature $\rho$ could double the magnet aperture, but is not included here.

Guessing that the ultimate choice would be many short bending magnets interspersed with many strong focusing magnets, the bend magnet occupancy is taken as $\eta=\frac{1}{2}$. Choosing a peak field $B=5 T$, the average is $\langle B\rangle=2.5 T$, and for $\langle B \rho\rangle \cong 50 T-m$ the bend radius becomes $\rho=20 m$. 
The entire concept includes diagnostics, correction devices, and an intricate geometry to position the various sets of beams around the chamber.
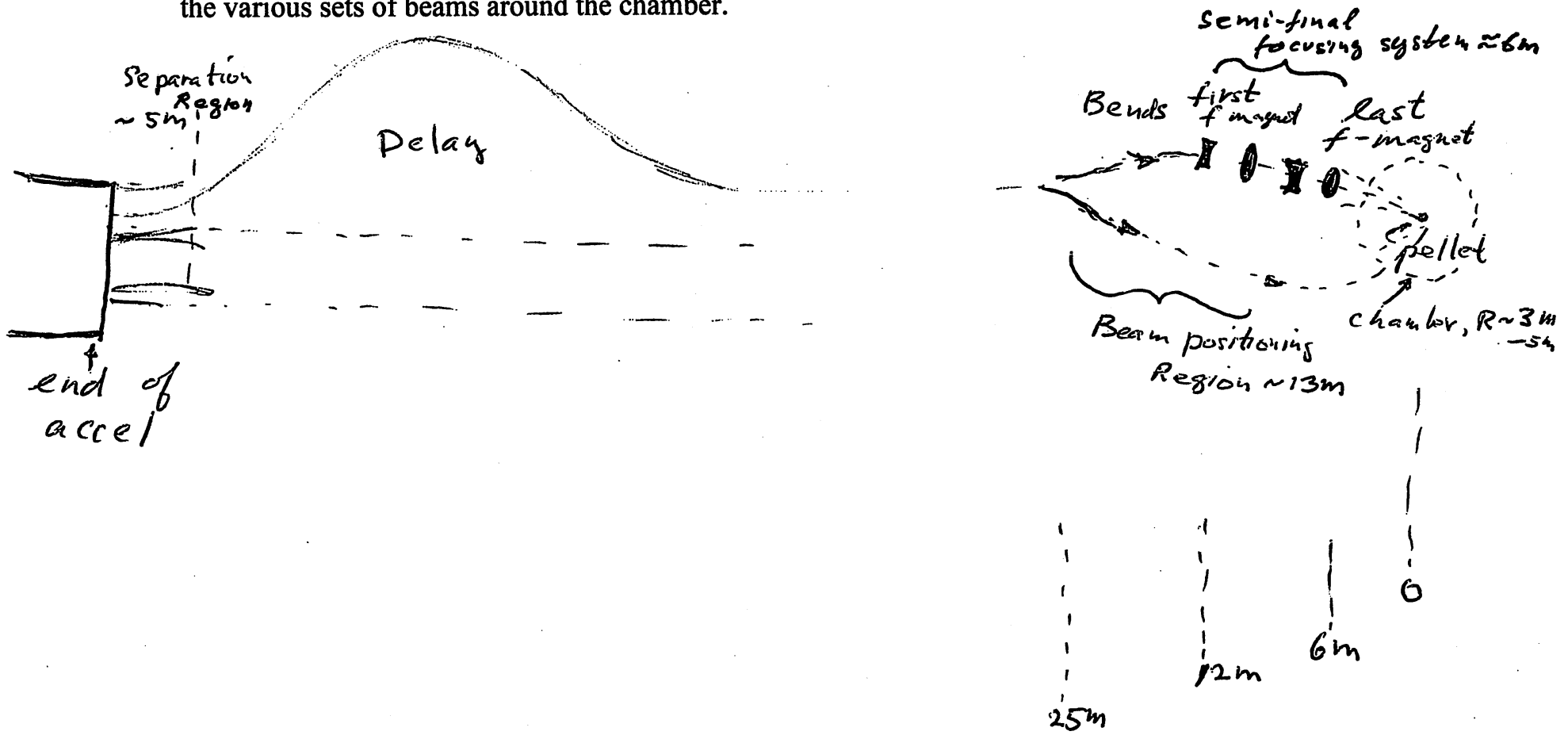

The aim is to transport as many of the beams as possible together in clusters, because of the favorable economics. The separation of the beams and individual reposition requires bends and quadrupoles around individual beams which make the total cluster geometry much bigger in that region than in the delay lines or the accelerator. For a place holder, let a distance of $25 \mathrm{~m}$ be devoted for all these manipulations, common to every set of beams. This means that these $25 \mathrm{~m}$ are not available for differential time delays. A similar but shorter interval is required at the end of the accelerator to separate the various groups of beams, which need different time delays. Let this distance be $5 \mathrm{~m}$. The total of $30 \mathrm{~m}$ is used for or included in the drift compression distance but excluded for delay adjustment purposes.

From

$$
\begin{gathered}
\Delta-21.6 m=(4)(120)(\theta-\sin \theta) \\
\theta-\sin \theta=0.27 \\
\theta \cong 1.2 \text { radians }
\end{gathered}
$$

and the total length of the four arcs is

$$
C=4 \rho \theta=(4)(20)(1.2)=96 m
$$

while the direct path is $c-21.6 \cong 74 \mathrm{~m}$.

Adding the 30 or so meters to take care of the separation of beams at the accelerator exit, the distribution of beams around the chamber, the semi-final focusing system, and the distance to the center of the chamber,

$$
\begin{array}{ll}
L_{D} & \text { igniter } \approx 126 \mathrm{~m} \\
L_{D} & \text { precursors } \cong 104 \mathrm{~m}
\end{array}
$$

and the transverse extent of the arced portion

$$
\Delta y=2(1-\cos \theta)(\rho)=25.5 \mathrm{~m}
$$


Adding the 30 or so meters to take care of the separation of beams at the accelerator exit, the distribution of beams around the chamber, the semi-final focusing system, and the distance to the center of the chamber,

$$
\begin{aligned}
& L_{D} \text { igniter } \approx 126 \mathrm{~m} \\
& L_{D} \text { precursors } \cong 104 \mathrm{~m}
\end{aligned}
$$

and the transverse extent of the arced portion

$$
\Delta y=2(1-\cos \theta)(\rho)=25.5 \mathrm{~m}
$$

\section{Longitudinal Emittance Requirement}

To focus at $126 \mathrm{~m}$ instead of the original $90 \mathrm{~m}$, the tilt is reduced to $( \pm 3 \%)\left(\frac{90}{126}\right)= \pm 2.1 \%$. At the longitudinal focus, the applied tilt and desired pulse duration of $50 \mathrm{ps}$ or $100 \mathrm{ps}$ give a full longitudinal phase area of

$$
\begin{gathered}
\mathrm{A}=\varepsilon_{\ell}=\Delta E \Delta t=\left(60 \times 10^{9} \mathrm{eV}\right)\left(4.2 \times 10^{-2}\right)\left(50 \text { or } 100 \times 10^{-12}\right) \\
\varepsilon_{\ell}=1.26 \times 10^{-1} \mathrm{eVs}
\end{gathered}
$$

or dividing by the charge state 12 ,

$$
1.05 \times 10^{-2} \text { volt seconds for the } 50 \mathrm{ps} \text { pulse }
$$
and $2.1 \times 10^{-2}$ volt second for the $100 \mathrm{ps}$ pulse.

At the $30 \mathrm{~ns}$ point at the exit of the accelerator

$$
\frac{\Delta V}{V}=\frac{2.1 \times 10^{-2}}{30 \times 10^{-9}} / 5 \times 10^{9}=1.4 \times 10^{-4}
$$

In the old designs $30 \mathrm{~ns}$ corresponded to approximately the pulse duration at the target and it was believed that achieving a $\frac{\Delta V}{V}=10^{-3}$ would be difficult. The $\mathrm{X}$-target concept requirement is therefore 7 times lower and more difficult to attain.

\section{The Beam Jog}

Approaching the reactor, it is necessary to relocate the beams such that they are situated on a cone with equal distance from the starting point.

From the side, this has the following appearance,

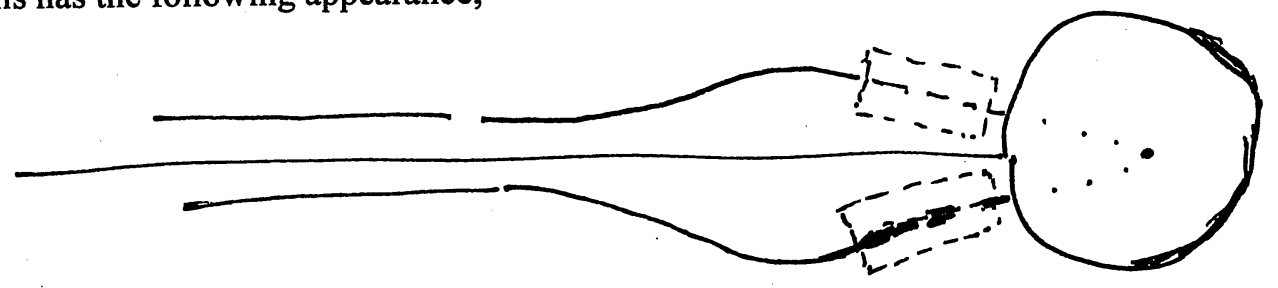

and axially,

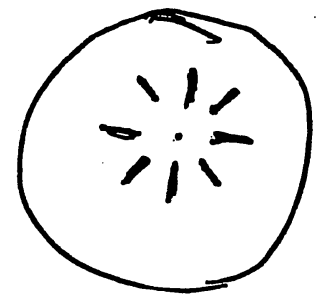


To change the principal planes on a FODO lattice, a possible solution is to recognize that midway between an $F$ and a $D$ the beams are circular

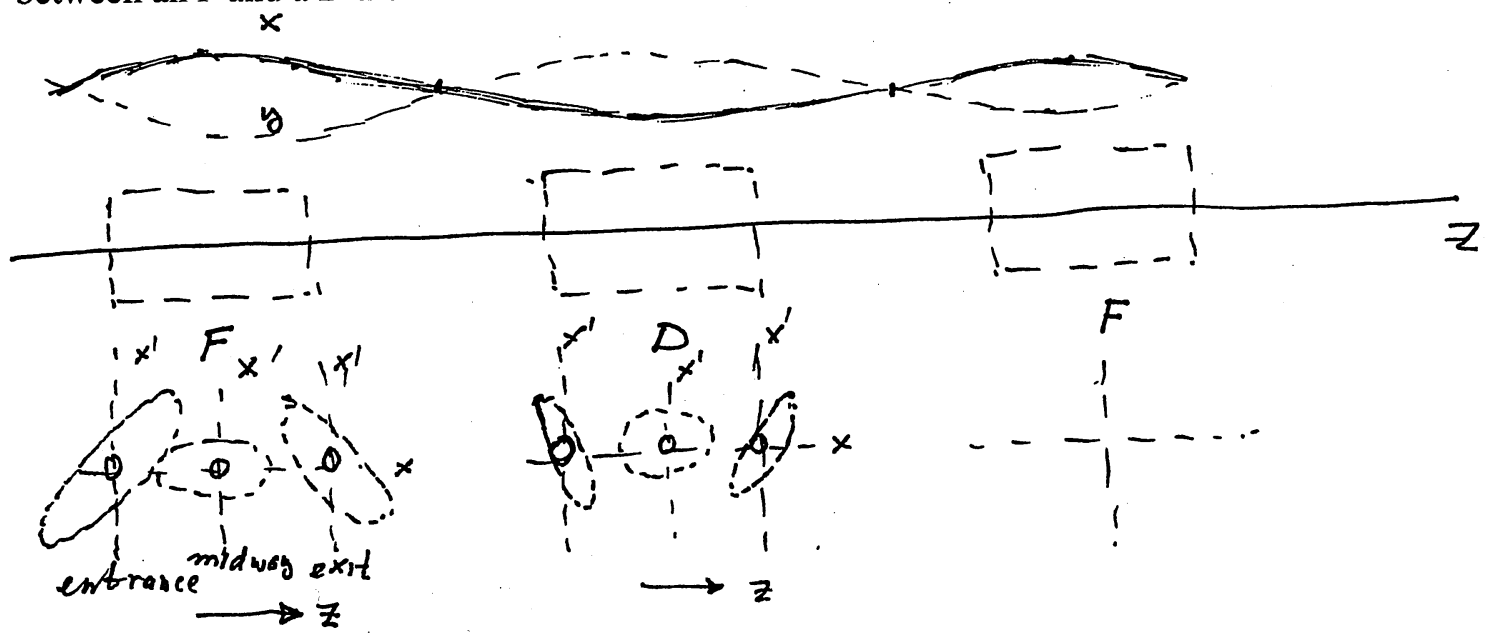

If at the midpoint a short half-strength quad is placed, then the resultant beam is round and with $x^{\prime}$ and $y^{\prime}$ zero. Very shortly after this, a new half quad, which is rotated in the desired new plane will rematch the beam into that plane

$$
\begin{aligned}
& \text { previous } \\
& \text { labtice }
\end{aligned}
$$

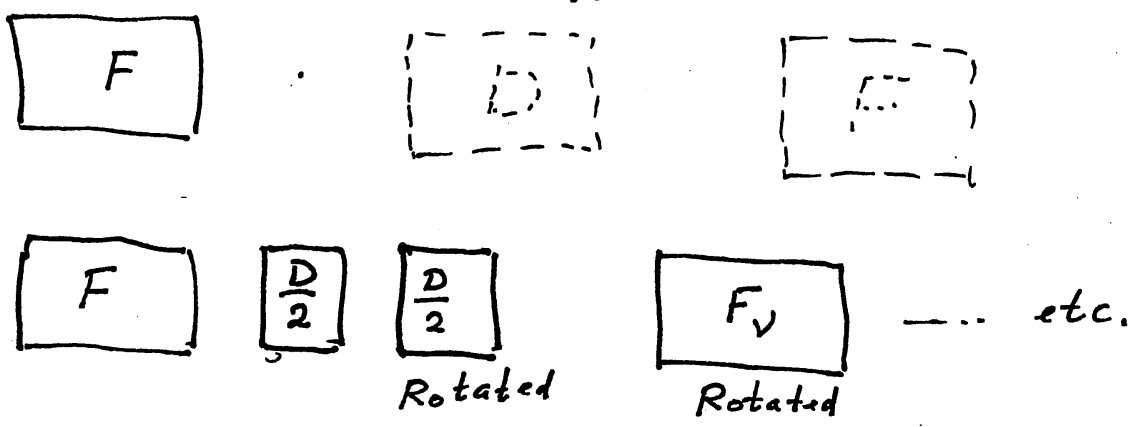

After this re-matching operation, it is necessary to have two symmetric bends, which displace the beam outward and give it the desired angle toward the center

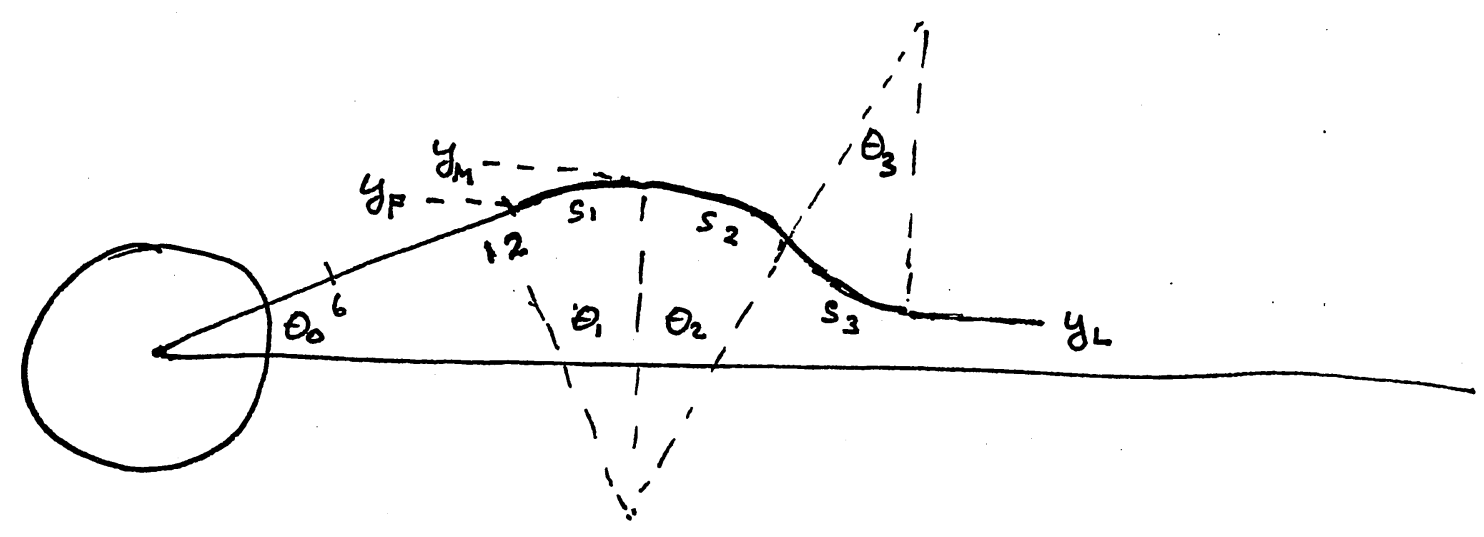


Taking a $6 \mathrm{~m}$ final or semifinal focusing section from the $6 \mathrm{~m}$ outer chamber wall distance, for a bundle convergence angle $\theta_{0}=\frac{1}{6}$, the distance from the axis $\mathrm{Y}_{F}=2 \mathrm{~m}$. Using the previous $\langle B \rho\rangle=50 \mathrm{Tm}$ and $\left\langle\mathrm{B}_{\text {bend }}\right\rangle=2.5 \mathrm{~T}$, for $\rho=20 \mathrm{~m}$, the arc $\mathrm{s}$, has $\theta_{0}=\theta$, and brings the beam parallel to the axis.

$$
s_{1}=\rho \theta,=(20)\left(\frac{1}{6}\right)=3.33 m
$$

The point $y_{m}$, the top of the arc, is $\rho\left(1-\cos \theta_{1}\right)=0.28 m$. Then $2 \rho\left(1-\cos \theta_{2}\right)=2 m$

$$
\theta_{2}=\theta_{3}=0.32, \quad s_{2}=s_{3}=6.4 m
$$

The position at the point where the trajectory is again parallel to the axis and at the right distance from it is $12 \mathrm{~m}$ (Chamber + Semifinal focus)

$$
\begin{aligned}
& 3.33 \mathrm{~m} s_{1} \\
& 6.4 \mathrm{~m} s_{2}+s_{3}
\end{aligned}
$$

$$
\overline{21.74 \mathrm{~m}}
$$

Assuming a distance of $4 \mathrm{~m}$ for the matching and re-matching section, a total of $\sim 26$ meters have been required for the beam manipulation near the chamber.

\section{References:}

(1) P.A. Seidl, E.P. Lee, R.O. Bangerter, A. Faltens, An Induction Linac Driver for A $0.44 \mathrm{MJ}$ Heavy-Ion Direct Drive Target, LBNL-3101E, February 8, 2010.

(2) Enrique Henestroza, B. Grant Logan, and L. John Perkins, Quasispherical fuel compression and fast ignition in a heavy-ion-driven $X$-target with one-sided illumination, PHYSICS OF PLASMAS 18, 032702 2011. http://pop.aip.org/resource/1/phpaen/v18/i3/p032702 s1 